\title{
Studies on X(4260) and X(4660) particles
}

\section{Meng Shi}

Department of Physics, Peking University, Beijing 100871, China

E-mail: shimeng1031@pku.edu.cn

\section{De-liang Yao}

Department of Physics, Peking University, Beijing 100871, China

E-mail: yaodeliang@pku.edu.cn

\section{Han-qing Zheng*}

Department of Physics, Peking University, Beijing 100871, China

E-mail: zhenghq@pku.edu.cn

Studies on the $\mathrm{X}(4260)$ and $\mathrm{X}(4660)$ resonant states in an effective lagrangian approach are reviewed. Using a Breit-Wigner propagator to describe their propagation, we find that the $\mathrm{X}(4260)$ has a sizable coupling to the $\omega \chi_{c 0}$ channel, while other couplings are found to be negligible. Besides, it couples much stronger to $\sigma$ than to $f_{0}(980):\left|g_{X \Psi \sigma}^{2} / g_{X \Psi f_{0}(980)}^{2}\right| \sim O(10)$. As an approximate result for $\mathrm{X}(4660)$, we obtain that the ratio of $\frac{\operatorname{Br}\left(X \rightarrow \Lambda_{c}^{+} \Lambda_{c}^{-}\right)}{\operatorname{Br}\left(X \rightarrow \Psi(2 s) \pi^{+} \pi^{-}\right)} \simeq 20$. Finally, taking $\mathrm{X}(3872)$ as an example, we also point out a possible way to extend the previous method to a more general one in the effective lagrangian approach.

Xth Quark Confinement and the Hadron Spectrum,

October 8-12, 2012

TUM Campus Garching, Munich, Germany

${ }^{*}$ Speaker. 
Many near threshold resonances, namely the $\mathrm{X}, \mathrm{Y}, \mathrm{Z}$ states, have been discovered in recent years experimentally [1], which has generated great interests in theoretical studies. For example, the $\mathrm{X}(3872)$ state is very close to $D^{0} D^{0 *}$ threshold, the $\mathrm{Y}(4260)$ state locates very close to the $\omega \chi_{c 0}$ and $D D_{1}(2420)$ thresholds, etc.. The understanding to these newly observed states makes a severe challenge to the study of the heavy quark spectrum.

In this talk we will review our recent studies of the $\mathrm{Y}(4260), \mathrm{Y}(4660)$ and $\mathrm{X}(3872)$ particles, using an effective lagrangian approach. We will also point out a possible way to extend the previous method to a more general one in the effective lagrangian approach. We hope the new proposal for future studies can be helpful in understanding the formation of hadronic molecule in the heavy quark system and in distinguishing between a molecular state and a heavy quarkonium state.

\section{On $\mathrm{X}(4260)$ state}

The X(4260) state was firstly discovered by BABAR Collaboration in 2005 [2] in initial state radiation (ISR) process, with $J / \psi \pi^{+} \pi^{-}$in the final state. The mass and width were found to be $M=4259 \pm 8(\text { stat. })_{-6}^{+2}($ sys. $) \mathrm{MeV}$ and $\Gamma=88 \pm 23(\text { stat. })_{-4}^{+6}($ sys. $) \mathrm{MeV}$, and the branching ratio was given by $\Gamma_{e^{+} e^{-}} \times B r\left(X \rightarrow \pi^{+} \pi^{-} J / \psi\right)=5.5 \pm 1.0_{-0.7}^{+0.8} \mathrm{eV}$. This state has also been confirmed by CLEO [3] and BELLE [4] experiments. On the other hand, it is puzzling that the X(4260) state is not found in the BES R-value measurement. Instead, there is only a dip structure in the energy region around $4.26 \mathrm{GeV}$ [5]. In theory aspect many theoretical works have been devoted to the study of the X(4260), and it is generally believed that the existence of the X(4260) signals a degree of freedom beyond conventional $\bar{c} c$ state. Many proposals have been made in the literature, e.g., charmonium, $\chi_{c 0} \rho^{0}$ molecule, $\omega \chi_{c 1}$ molecule, $c \bar{c} g$ hybrid state, $\Lambda_{c} \bar{\Lambda}_{c}$ bayronium, $D_{1} \bar{D}$ or $D_{0} \bar{D}_{0}^{*}$ molecule, etc.. There even exists the suggestion that the $\mathrm{X}(4260)$ may not even be a resonant state [6]. In the following we will however assume that $\mathrm{X}(4260)$ is a propagating Breit-Wigner state and the denominator of the $\mathrm{X}(4260)$ is parameterized as,

$$
D_{X}\left(q^{2}\right)=M_{X}^{2}-q^{2}-i \sqrt{q^{2}}\left(g_{1} k_{1}+g_{2} k_{2}+\Gamma\left(q^{2}\right)+\Gamma_{0}\right),
$$

where $M_{X}$ is the bare mass of the Breit-Wigner particle, $g_{1}\left(g_{2}\right)$ denotes the coupling of $\mathrm{X}(4260)$ to the nearest channel below (above) the pole position, $k_{1}$ and $k_{2}$ are corresponding channel momentum respectively. $\Gamma\left(q^{2}\right)$ denotes the partial decay width to $J / \Psi \pi \pi$ and the constant width $\Gamma_{0}$ simulates other possible decay channels apart from those included explicitly in Eq. (1.1). Most likely $\Gamma_{0}$ would represent the (missing) open charm channels which are unobserved experimentally.

We write down effective lagrangians describing its photoproduction and decay into $J / \Psi \pi \pi$ states:

$$
\begin{aligned}
\mathscr{L}_{\gamma X} & =g_{0} X_{\mu \nu} F^{\mu v} \\
\mathscr{L}_{X \psi P P} & =h_{1} X_{\mu \nu} \psi^{\mu v}<u_{\alpha} u^{\alpha}>+h_{2} X_{\mu \nu} \psi^{\mu v}<\chi_{+}>+h_{3} X_{\mu \alpha} \psi^{\mu \beta}<u_{\beta} u^{\alpha}>
\end{aligned}
$$

The above lagrangian obeys chiral symmetry up to $\mathscr{O}\left(p^{2}\right)$ level and for more detailed explanation we refer to Ref. [7]. Eq. (1.2) is not enough yet to appropriately describe the strong interactions of the I=0 $s$-wave $\pi \pi$ final state. We therefore only use Eq. (1.2) to calculate the tree level decay 
amplitude $\mathscr{A}^{\text {tree }}$, and further improve the calculation by making use of the couple channel final state theorem [8]:

$$
\begin{aligned}
& \mathscr{A}_{1}=\mathscr{A}_{1}^{\text {tree }} \alpha_{1}(s) T_{11}(s)+\mathscr{A}_{2}^{\text {tree }} \alpha_{2}(s) T_{21}(s), \\
& \mathscr{A}_{2}=\mathscr{A}_{1}^{\text {tree }} \alpha_{1}(s) T_{12}(s)+\mathscr{A}_{2}^{\text {tree }} \alpha_{2}(s) T_{22}(s),
\end{aligned}
$$

where the subscripts 1,2 denote the $\pi \pi$ and $\bar{K} K$ channels, respectively, and $\alpha_{i}$ are mild polynomials to be determined by fit. For the $\pi \pi, \bar{K} K$ scattering $T$ matrix we chose three solutions found in the literature: Padé [9], K-matrix [10], PKU [11]. The fit results are shown in Fig. 1 and table 1. From numerical studies we draw the following observations: A large coupling between $\mathrm{X}(4260)$ and $\omega \chi_{c 0}$ is obtained, while other couplings are found to be negligible; It is estimated that the $\mathrm{X}$ particle couples much stronger to $\sigma$ than to $f_{0}(980):\left|g_{X \Psi \sigma}^{2} / g_{X \Psi f_{0}(980)}^{2}\right| \sim O(10)$. The value
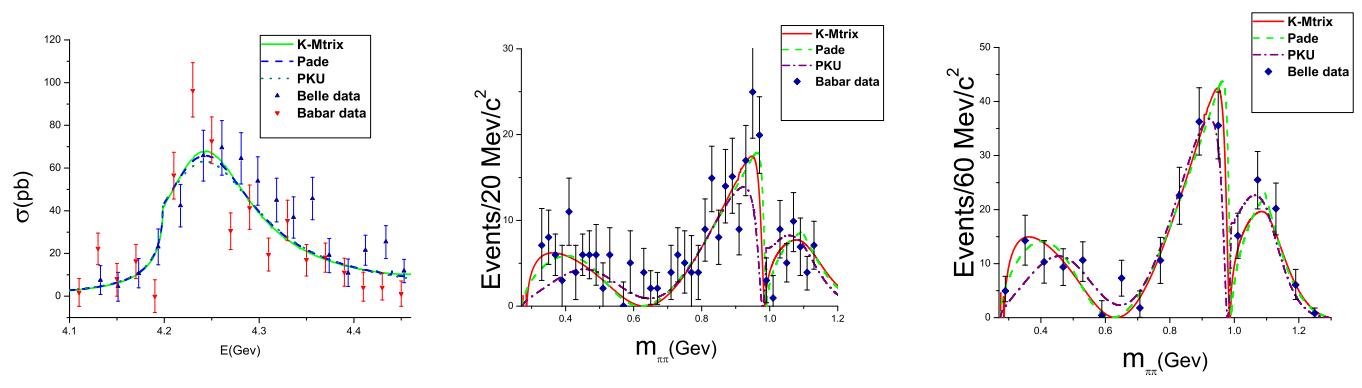

Figure 1: Left, the $J / \Psi \pi \pi$ cross section from BABAR[2] and BELLE[4]; middle, the $\pi \pi$ invariant mass spectrum from BABAR[2]; right, the $\pi \pi$ invariant mass spectrum from BELLE[4].

of $g_{0}$ given in table 1 corresponds to $\Gamma\left(e^{+} e^{-}\right)=228 \mathrm{eV}$. These numbers are in reasonable range comparing with the BES bound given in Ref. [12].

One unresolved puzzle with respect to $\mathrm{X}(4260)$ is that, if it is a $\bar{c} c$ state, it is hard to explain the absence of its decay into open charm channels, e.g., $D \bar{D}, D^{*} \bar{D}$, etc.. Here in the fit the constant width is found to be $50 \mathrm{MeV}$ with sizable uncertainty. One possible explanation to this puzzle is that there exists a cancelation between contributions from $\gamma^{*}$ and $X$ to open charms. We find two

\begin{tabular}{cc}
\hline \hline & Fit I (Padé) \\
\hline$\chi_{\text {d.o.f }}^{2}$ & $\frac{108.9}{93-14}$ \\
$g_{0}(\mathrm{MeV})$ & $9.984 \pm 1.046$ \\
$g_{1}$ & $0.608 \pm 0.094$ \\
$M_{X}(\mathrm{GeV})$ & $4.263 \pm 0.010$ \\
$\Gamma_{0}(\mathrm{GeV})$ & $0.051 \pm 0.008$ \\
\hline \hline
\end{tabular}

Table 1: Fit results assuming X(4260) couples to $\omega \chi_{c 0} ; \Gamma_{0}$ and background included in the fit.

\begin{tabular}{cc}
\hline \hline & Fit I (Padé) \\
\hline$\chi_{\text {d.o.f }}^{2}$ & $\frac{147.0}{93-14}$ \\
$g_{0}(\mathrm{MeV})$ & $6.836 \pm 0.245$ \\
$g_{1}$ & $0.514 \pm 0.008$ \\
$M_{X}(\mathrm{GeV})$ & $4.2118 \pm 0.012$ \\
$\Gamma_{0}(\mathrm{GeV})$ & $0.017 \pm 0.014$ \\
\hline \hline
\end{tabular}

Table 2: Fit results assuming an equal X(4260) coupling to $\omega \chi_{c 0}$ and $\bar{D} D_{1} ; \Gamma_{0}$ and background included in the fit.

poles located at $\sqrt{s}=4177.3-90.0 i \mathrm{MeV}$ on sheet III and $\sqrt{s}=4.227 .4-39.7 i \mathrm{MeV}$ on sheet IV respectively. 
One possible explanation found in the literature is that the $\mathrm{X}(4260)$ be a candidate of $c \bar{c} g$ hybrid state. In such a situation, the $\mathrm{X}(4260)$ may couple strongly to $D D_{1}$ channel [13] which is however not supported by our fit. In table 2, we list the result by enforcing a equal coupling of $X \omega \chi_{c 0}$ and $X D D_{1}$. Comparing with the fit result in table 1 the total $\chi^{2}$ is increased considerably.

Through our numerical studies, we also found that, except for the $X \omega \chi_{c 0}$ coupling, there are no signals for the $\mathrm{X}(4260)$ coupling to other channels. Hence we exclude most of the molecular assignment to $\mathrm{X}(4260)$. Assuming the occurrence of the cancelation between $\gamma^{*}$ and $\mathrm{X}(4260)$ to open charm channels, we may conclude from our numerical analysis that the $\mathrm{X}(4260)$ is mainly a $c \bar{c}$ state renormalized by the $\omega \chi_{c 0}$ continuum. Our estimated $\Gamma_{e^{+} e^{-}} \simeq 228 \mathrm{eV}$, which is within the upper limit set up from BES experiments [12]. The renormalization effect due to $\omega \chi_{c 0}$ loop should be important because a naive quark model calculation tends to give a large value of $\Gamma_{e^{+}} e^{-}$. A screening inter-quark potential can lower the mass of $4^{3} S_{1}$ state down to $4273 \mathrm{MeV}$ with a $\Gamma_{e^{+} e^{-}} \simeq 970 \mathrm{eV}$, S-D mixing may reduce this number by half [14], which is however still not small enough in comparison with our estimate. Hence a sizable mixing with the continuum is crucial in reducing the leptonic decay width. Notice that the $\gamma^{*}-\mathrm{X}$ transition coupling $g_{0}$ obeys

$$
g_{0}^{R}=Z_{X}^{1 / 2} g_{0}^{B}
$$

where $g_{0}^{B}$ denotes the value of $g_{0}$ at tree level - value obtained from simple potential model calculation without considering the continuum mixing, and $g_{0}^{R}$ is the 'renormalized' quantity measured by experiments. The wave function renormalization constant $Z_{X}$ is finite and calculable for s-wave interaction in non-relativistic limit. To understand this better let us consider a simplified situation when $\mathrm{X}$ is a bound state with respect to the $\omega \chi_{c 0}$ channel then

$$
Z_{X}=\frac{1}{1-\operatorname{Re} \Sigma^{\prime}\left(\mu^{2}\right)} \simeq \frac{1}{1+\frac{g_{1}}{2 \sqrt{2}} \sqrt{\frac{m_{R}}{\varepsilon}}},
$$

where we have let $\mu=M_{t h}-\varepsilon$ and $\varepsilon$ is the binding energy, $m_{R}=\frac{M_{\chi} m_{\omega}}{M_{\chi}+m_{\omega}}=637 \mathrm{MeV}$. The loop correction leads to a reduction of the 'tree level' value of $\Gamma_{e^{+} e^{-}}$by a factor $Z_{X}$.

\section{A brief comment on $X(4660)$}

\begin{tabular}{ccc}
\hline \hline & fit I (Padé) & fit II (K-matrix) \\
\hline$\chi_{\text {d.o.f }}^{2}$ & 1.38 & 1.00 \\
$g_{0}(\mathrm{MeV})$ & $7.118 \pm 0.633$ & $7.025 \pm 0.630$ \\
$g_{1}$ & $2.155 \pm 0.273$ & $2.103 \pm 0.275$ \\
$M_{X}(\mathrm{GeV})$ & $4.659 \pm 0.011$ & $4.652 \pm 0.010$ \\
\hline \hline
\end{tabular}

Table 3: Parameters given by fit to X(4660) data.

The X(4660) state is observed to decay into $\Lambda_{c} \bar{\Lambda}_{c}$ and $\psi(2 s) \pi \pi$. [15] The effective lagrangian describing the $\mathrm{X}(4660)$ interaction are the following:

$$
\mathscr{L}_{X \gamma}=g_{0} F_{X \mu \nu} F_{\gamma}^{\mu \nu}
$$




$$
\begin{aligned}
& \mathscr{L}_{\Psi^{\prime} X p p}=h_{1} F_{X \mu v} F_{\Psi^{\prime}}^{\mu v}<u_{\rho} u^{\rho}>+h_{2} F_{X \mu v} F_{\Psi^{\prime}}^{\mu v}<\chi_{+}>+h_{3} F_{X \mu \alpha} F_{\Psi^{\prime}}^{\mu \beta}<u_{\beta} u^{\alpha}> \\
& \mathscr{L}_{X \Lambda_{c}^{+} \Lambda_{c}^{-}}=g_{1} \bar{\Psi}_{c} \gamma^{\mu} \Psi_{c} X_{\mu} .
\end{aligned}
$$

The denominator of the Breit-Wigner $\mathrm{X}(4660)$ propagator is parameterized as

$$
D_{X}\left(q^{2}\right)=q^{2}-M_{X}^{2}+i \sqrt{q^{2}}\left(\frac{3}{2} \Gamma_{\psi(2 S) \pi^{+} \pi^{-}}\left(q^{2}\right)+2 \Gamma_{\psi(2 S) K^{+} K^{-}}\left(q^{2}\right)+\Gamma_{\Lambda_{c}^{+} \Lambda_{c}^{-}}\left(q^{2}\right)\right) .
$$

Final state interactions among $\pi \pi$ and $\bar{K} K$ are also taken into account. The fit results are listed in table 3. There are also two poles lying on the third and fourth Riemann sheet: $\sqrt{s}=4618.5-$ $73.5 i \mathrm{MeV}$ (III) and $\sqrt{s}=4623.3-68.3 i \mathrm{MeV}$ (IV) for Padé method; $\sqrt{s}=4616.2-69.1 i \mathrm{MeV}$ (III) and $\sqrt{s}=4624.0-60.7 i \mathrm{MeV}$ (IV) for K-matrix method.

The ratio of $\frac{B r\left(X \rightarrow \Lambda_{c}^{+} \Lambda_{c}^{-}\right)}{\operatorname{Br}\left(X \rightarrow \Psi(2 s) \pi^{+} \pi^{-}\right)}$are estimated as 23.9 for Padé method and 19.3 for K-Matrix method and the results are comparable to those in Refs. $[16,17]$. We point out that the value of $g_{0}$ given in table 3 corresponds to $\Gamma_{e^{+} e^{-}} \simeq 102 \mathrm{eV}$. Assuming the magnitude of

$$
\Gamma_{e^{+} e^{-}} \times \operatorname{Br}\left(X \rightarrow \Psi(2 S) \pi^{+} \pi^{-}\right) \approx 5 e V,
$$

which will be similar with $\mathrm{X}(4260)$ [2], the branch ratio of $\operatorname{Br}\left(X \rightarrow \Psi(2 S) \pi^{+} \pi^{-}\right) \approx 5 \%$ can be derived. This means $\operatorname{Br}\left(X \rightarrow \Lambda_{c}^{+} \Lambda_{c}^{-}\right) \approx 95 \%$, in agreement with the ratio $\frac{B r\left(X \rightarrow \Lambda_{c}^{+} \Lambda_{c}^{-}\right)}{\operatorname{Br}\left(X \rightarrow \Psi(2 s) \pi^{+} \pi^{-}\right)} \approx 20$.

\section{Future improvement and outlook}
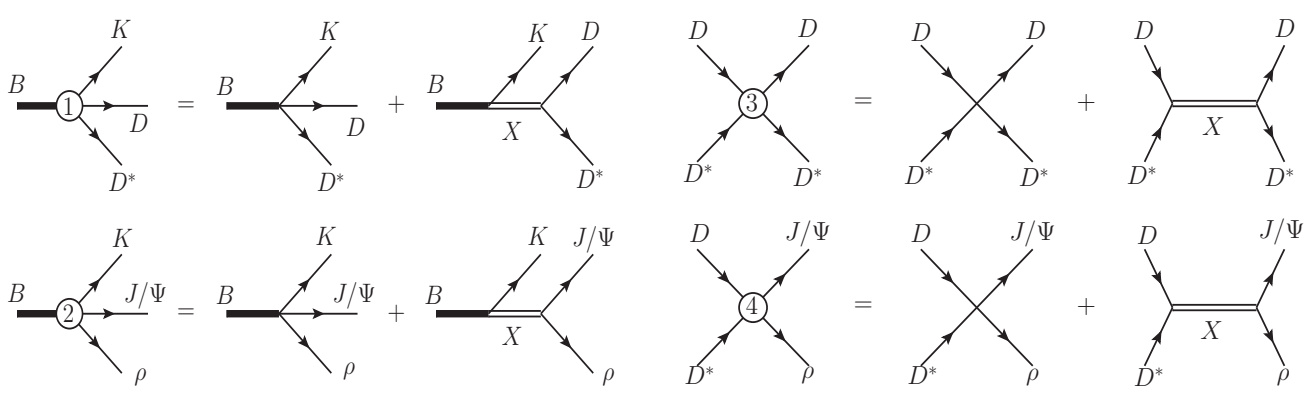

Figure 2: Vertices appeared when studying X(3872).

In above we have performed a numerical analysis on the near threshold resonances X(4260) and X(4660). However, one of the key assumption we implicitly made in our analysis is that they propagate as a particle, i.e., a Breit-Wigner propagator is used to describe their propagation. This assumption can in principle be examined and tested by including more complicated dynamics. Taking the $\mathrm{X}(3872)$ particle for example, as shown in figures $2-3$, one can include the final state interactions between $D^{*} \bar{D}$ and sum up the bubble chains. The resummation of the diagrams shown in figures 2 and 3 is an approximation but becomes exact in the non-relativistic limit. In this situation a molecular type state may be generated from the bubble chain. Such a pole might interact with the bare Breit-Wigner particle and the final physical picture might be then determined by the competition of the two different type of poles. A study along this direction is underway.

Acknowledgements: This work is supported in part by National Nature Science Foundations of China under contract number 10925522 and 11021092. 


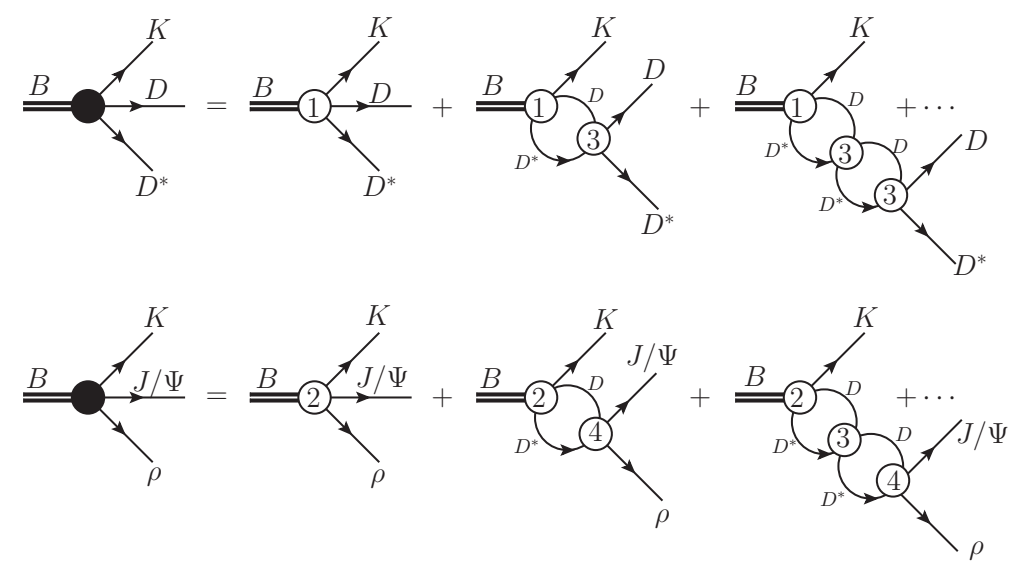

Figure 3: The sum of bubble diagrams.

\section{References}

[1] J. Beringer et al. (Particle Data Group), Phys. Rev. D86,010001(2012).

[2] B. Aubert et al. (BABAR Collaboration), Phys. Rev. Lett. 95, 142001 (2005).

[3] T. E. Coan et al. (CLEO Collaboration), Phys. Rev. Lett. 96, 162003 (2006).

[4] C. Z. Yuan et al. (BELLE Collaboration), Phys. Rev. Lett. 99, 182004 (2007).

[5] M. Ablikim et al. (BES Collaboration), Phys. Lett. B 660, 315 (2008).

[6] D. Y. Chen, J. He, and X. Liu, Phys. Rev. D 83, 054021 (2011).

[7] L. Y. Dai, M. Shi, G. -Y. Tang and H. Q. Zheng, arXiv:1206.6911 [hep-ph].

[8] K. L. Au, D. Morgan and M. R. Pennington, Phys. Rev. D 351633 (1987); D. Morgan and M. R. Pennington, Phys. Rev. D 481185 (1993).

[9] L. Y. Dai, X. G. Wang and H. Q. Zheng, Commun. Theor. Phys. 57, 841 (2012).

[10] Y. Mao, X. G. Wang, O. Zhang, H. Q. Zheng, and Z. Y. Zhou, Phys. Rev. D 79116008 (2009).

[11] Z. Y. Zhou et al., JHEP 0502, 043 (2005).

[12] X. H. Mo et al., Phys. Lett. B 640, 182 (2006).

[13] F. E. Close, P. R. Page, Phys. Lett. B 628, 215 (2005).

[14] B. Q. Li, K. T. Chao, Phys. Rev. D 79, 094004 (2009).

[15] X. L. Wang et al, Phys. Rev. Lett. 99, 142002 (2007); B. Aubert et al, Phys. Rev. Lett. 98, 212001 (2007); G. Pakhlova et al, Phys. Rev. Lett. 101, 172001 (2008).

[16] G. Cotugno, R. Faccini, A. D. Polosa, and C. Sabelli. Phys. Rev. Lett. 104, 132005 (2010)

[17] F. K. Guo, J. Haidenbauer, C. Hanhart, U. G. Meissner, arXiv:1005.2055 [hep-ph]. 